TRANSACTIONS OF THE

AMERICAN MATHEMATICAL SOCIETY

Volume 349, Number 2, February 1997, Pages 789-807

S 0002-9947(97)01726-1

\title{
LIMITING SUBHESSIANS, LIMITING SUBJETS AND THEIR CALCULUS
}

\author{
ALEXANDER D. IOFFE AND JEAN-PAUL PENOT
}

\begin{abstract}
We study calculus rules for limiting subjets of order two. These subjets are obtained as limits of sequences of subjets, a subjet of a function $f$ at some point $x$ being the Taylor expansion of a twice differentiable function which minorizes $f$ and coincides with $f$ at $x$. These calculus rules are deduced from approximate (or fuzzy) calculus rules for subjets of order two. In turn, these rules are consequences of delicate results of Crandall-Ishii-Lions. We point out the similarities and the differences with the case of first order limiting subdifferentials.
\end{abstract}

\section{INTRODUCTION}

The interest in second order nonsmooth analysis seems to be growing in recent years, motivated both by applications and by analysis itself. In the classical calculus there are two possibilities for defining second order derivatives: as the derivative of the first order derivative or by means of the 2-jet, i.e. the second order Taylor expansion. As we know, the two definitions are not equivalent unless the function or the map is twice continuously differentiable. Not surprisingly, we should expect different results from extensions of these two approaches to nonsmooth settings.

The objects to be studied in this paper pertain to the second approach mentioned above. Moreover, they possess a unilateral (or one sided) feature which is characteristic of most nonsmooth concepts. In fact these objects are the second order generalized subjets introduced in [4], [5]. Our purpose is to develop a calculus of these generalized 2-subjets comparable to what we have for first order subdifferentials, more specifically, for "approximate" subdifferentials studied geometrically by Mordukhovich [14], [15], and analytically by Ioffe [9] and Kruger [12]. We follow here the analytic pattern which includes first a development of a "fuzzy" calculus of "Fréchet 2-subjets" similar to first order Fréchet or Dini subdifferentials, and then obtain calculus rules by passing to the limit (cf. [9], [10]).

It turns out, however, that at each of the two steps we run into technical difficulties which have no analogues in the first order theory. At the "fuzzy calculus" stage the penalization technique similar to what has been used in the first order case does not by itself provide the separation of variables needed for subdifferentiation of various "composite" functions. At the "passage to limit" step the difficulty is caused by the fact that Fréchet 2-subjets are always unbounded.

Received by the editors August 3, 1994 and, in revised form, September 5, 1995.

1991 Mathematics Subject Classification. Primary 28A15, 46G05; Secondary 26A24, 26A27.

The first author's research was supported in part by the U.S.-Israel Binational Science Foundation, under grant no. 90-00455. 
At the first step the difficulty is overcome with the help of a decomposition lemma of Crandall-Ishii-Lions [5] relying on some work of Jensen [11]; at the second step it is overcome with the help of a notion of "efficient subjet", which allows us to split a converging part off from a sequence of subjets going to infinity and to define a second order analogue of singular differentials. All this makes the second order calculus substantially more complicated technically than the first order calculus, but, as a result, the main formulae for subdifferentiation of a sum and a composition receive second order counterparts. This fact paves the way for applications which are not considered here.

\section{DEFinitions AND ELEMENTARY PROPERTIES}

Throughout the paper $X, Y, Z$ denote the real finite-dimensional Euclidean spaces and $A, B, C$ linear operators between them (or their matrices). We use the following notation: $L(X, Y)$ denotes the space of linear operators from $X$ into $Y ; L_{s}(X)$ stands for the space of symmetric linear operators in $X$ and $L_{s}^{+}(X)$ for the cone of positive semidefinite elements of $L_{s}(X)$. The inner product is denoted by $(u \mid v)$, and its associated norm is $\|x\|=(x \mid x)^{1 / 2}$, whatever the space is. The unit sphere is the set $S=\{x:\|x\|=1\}$.

On the space $L_{s}(X)$ we consider a norm $A \mapsto\|A\|$ satisfying the following monotonicity property:

$$
B \geq A \geq 0 \Rightarrow\|B\| \geq\|A\|,
$$

where $A \geq 0$ means that $(A v \mid v) \geq 0$ for all $v$. Among the norms satisfying this property are the supremum norm given by

$$
\|A\|=\max _{v \in S}|(A v \mid v)|=\max \left(\left|\lambda_{1}\right|, \ldots,\left|\lambda_{n}\right|\right),
$$

where $\lambda_{1}, \ldots, \lambda_{n}$ are the eigenvalues of $A$, and the Frobenius norm given for $A=$ $\left(a_{i, j}\right)$ by

$$
\|A\|:=\sqrt{\sum_{i, j=1, \ldots, n} a_{i, j}^{2}}=\sqrt{\operatorname{tr}\left(A^{2}\right)} .
$$

For $A \in L_{s}(X), B \in L_{s}(Y), C \in L(X, Y)$ we set

$$
Q(A, B, C)=\left(\begin{array}{cc}
A & C^{*} \\
C & B
\end{array}\right) \in L_{s}(X \oplus Y) .
$$

As a rule we consider extended-real-valued functions, and for such a function $f$ we set

$$
\operatorname{dom} f=\{x: f(x)<\infty\} .
$$

Definition 2.1. Given $x \in \operatorname{dom} f$, the set $\partial_{-}^{2} f(x)$ of all pairs $\left(x^{*}, A\right) \in X \times L_{s}(X)$ with the property

$$
\liminf _{\|v\| \rightarrow 0}\|v\|^{-2}\left(f(x+v)-f(x)-\left(x^{*} \mid v\right)-\frac{1}{2}(A v \mid v)\right) \geq 0
$$

is called the Fréchet second order subjet of $f$ at $x$.

The set

$$
\partial_{-}^{2} f\left(x, x^{*}\right)=\left\{A \in L_{s}(X):\left(x^{*}, A\right) \in \partial_{-}^{2} f(x)\right\}
$$

is the set of Fréchet subhessians of $f$ at $\left(x, x^{*}\right)$ (cf. [17]). It is a closed convex subset of $L_{s}(X)$, as we can easily see. 
The preceding definition contains a slight abuse of language since traditionally the 2-jet of a twice differentiable function $f$ at $x$ is the triple $\left(f(x), D f(x), D^{2} f(x)\right)$ of $\mathbb{R} \times X^{*} \times L_{s}^{2}(X, \mathbb{R})$ or its image $\left(f(x), \nabla f(x), \nabla^{2} f(x)\right)$ in $\mathbb{R} \times X \times L_{s}(X)$ through the usual identification, and its 2 -subjet should be

$$
J_{-}^{2} f(x)=\left\{\left(f(x), x^{*}, A\right):\left(x^{*}, A\right) \in \partial_{-}^{2} f(x)\right\} .
$$

To place the objects just introduced in proper perspective we recall the wellknown notions of (first order) Fréchet subdifferential, (lower or) contingent derivative, and lower second order epiderivative (see [1], [9], [10], [20]).

The first is defined as the set $\partial_{-} f(x)$ of all $x^{*}$ with the property that

$$
\liminf _{\substack{v \rightarrow 0 \\ v \neq 0}}\|v\|^{-1}\left(f(x+v)-f(x)-\left(x^{*} \mid v\right)\right) \geq 0,
$$

the second as

$$
f_{-}^{\prime}(x, v):=\liminf _{(t, u) \rightarrow\left(0_{+}, v\right)} t^{-1}(f(x+t u)-f(x)),
$$

and the third as

$$
f_{-}^{\prime \prime}\left(x, x^{*}, v\right)=\liminf _{(t, u) \rightarrow\left(0_{+}, v\right)} 2 t^{-2}\left(f(x+t u)-f(x)-t\left(x^{*} \mid u\right)\right) .
$$

Proposition 2.1. Let $f$ be lower semicontinuous (l.s.c.) near $x$ and finite at $x$. Then the following four properties are equivalent:

(a) $\left(x^{*}, A\right) \in \partial^{2} f(x)$;

(b) for any $\varepsilon>0$ the function

$$
v \mapsto f(x+v)-f(x)-\left(x^{*} \mid v\right)-\frac{1}{2}(A v \mid v)+\varepsilon\|v\|^{2}
$$

has a local minimum at zero;

(c) $x^{*} \in \partial_{-} f(x)$ and $(A v \mid v) \leq f_{-}^{\prime \prime}\left(x, x^{*}, v\right), \forall v \in X$;

(d) $x^{*} \in \partial_{-} f(x)$ and $(A v \mid v) \leq f_{-}^{\prime \prime}\left(x, x^{*}, v\right)$, for any $v$ such that $\left(x^{*} \mid v\right)=f_{-}^{\prime}(x, v)$.

The proof is not difficult and is left to the reader.

In the next section we shall see that second order Fréchet subjets have analytic properties similar to (first order) Fréchet subdifferentials, and now we proceed with the definition of the basic limiting objects.

Definition 2.2. Let $f$ be lower semicontinuous near $x$ and finite at $x$. The approximate or limiting 2-subjet of $f$ at $x$ is defined as

$$
\bar{\partial}^{2} f(x)=\limsup _{\substack{\mathfrak{f} \rightarrow x \\ \text { s. }}} \partial_{-}^{2} f(u),
$$

where lim sup is understood in the classical sense of Painlevé-Kuratowski, and $u \stackrel{f}{\rightarrow}$ $x$ means " $u \rightarrow x$ and $f(u) \rightarrow f(x)$ ".

We also set

$$
\bar{\partial}^{2} f\left(x, x^{*}\right)=\left\{A \in L_{s}(X):\left(x^{*}, A\right) \in \bar{\partial}^{2} f(x)\right\}
$$

and call this set the set of approximate or limiting subhessians of $f$ at $\left(x, x^{*}\right)$.

An interpretation of $\bar{\partial}^{2} f(x)$ can be given by using the graph $J_{-}^{2} f$ of the multimapping $x \rightsquigarrow J_{-}^{2} f(x)$ given by

$$
J_{-}^{2} f=\left\{\left(x, f(x), x^{*}, A\right):\left(x^{*}, A\right) \in \partial_{-}^{2} f(x)\right\} ;
$$


one has

$$
\bar{\partial}^{2} f(x)=\left\{\left(x^{*}, A\right):\left(x, f(x), x^{*}, A\right) \in \operatorname{cl} J_{-}^{2} f\right\},
$$

where cl $S$ denotes the closure of the set $S$.

To finish the introduction of our basic concepts we extend the last definition to mappings between spaces $X$ and $Y$.

Definition 2.3. Let $F$ be a continuous map from $X$ into $Y$ defined in a neighborhood of $x$. For any $y^{*} \in Y^{*}$ we set

$$
\bar{D}^{2} F\left(y^{*}, x\right)=\limsup _{\left(u, z^{*}\right) \rightarrow\left(x, y^{*}\right)} \partial_{-}^{2}\left(z^{*} \circ F\right)(u)
$$

and call the set-valued map $y^{*} \rightarrow \bar{D}^{2} F\left(y^{*}, x\right)$ the (approximate, or limiting) second order cojet of $F$ at $x$.

The first order analogue of this object coincides with the coderivative introduced by Aubin and Mordukhovich (see [1], [15]), which is obtained by subdifferentiation of the indicator function of the graph of $F$ and extends to set-valued maps as well. The other type of ("scalarized") coderivatives was studied by Ioffe in [9]. The scalarization formula proved in [9] states that both derivatives coincide for Lipschitzian mappings between finite-dimensional spaces. Otherwise they can differ. We do not discuss here possible second order extensions of the second scheme (which can be obtained from Definition 2.3 if we keep $z^{*}$ equal to $\left.y^{*}\right)$.

\section{3. "FuZzY" CALCULUS OF FrÉCHET SUBJETS}

In this section we develop a "fuzzy" calculus for Fréchet 2-subjets similar to that developed for first order Dini-Hadamard and Fréchet subdifferentials in [9], [10]. It will consist of two basic theorems, one for a sum and another one for a composition.

Theorem 3.1. Let the functions $f_{1}, \ldots, f_{k}$ be lower semicontinuous (l.s.c.) near $x$ and finite at $x$. Set $f=f_{1}+\cdots+f_{k}$ and assume that $\left(x^{*}, A\right) \in \partial_{-}^{2} f(x)$. Then for any $\varepsilon>0$ there are $k$ triples $\left(x_{i}, x_{i}^{*}, A_{i}\right), i=1, \ldots, k$, such that, for $i=1, \ldots, k$, $\left(x_{i}^{*}, A_{i}\right) \in \partial_{-}^{2} f\left(x_{i}\right),\left|f_{i}\left(x_{i}\right)-f_{i}(x)\right|<\varepsilon,\left\|x_{i}-x\right\|<\varepsilon$, and

$$
\left\|x_{1}^{*}+\cdots+x_{k}^{*}-x^{*}\right\|<\varepsilon, \quad A_{1}+\cdots+A_{k} \geq A-\varepsilon I .
$$

If moreover the functions $f_{1}, \ldots, f_{n}$ are convex, we may assume that $A_{i} \geq 0$.

Changing $A_{i}$ into $A_{i}-\frac{1}{k}\left(\sum_{j=1}^{k} A_{j}-A+\varepsilon I\right)$, we may write the last relation in the equality form:

$$
A_{1}+\cdots+A_{k}=A-\varepsilon I \text {. }
$$

The proof of the theorem is based on the following results.

Lemma 3.2 ([5, Theorem 3.2]). Suppose that $\varphi_{1}, \ldots, \varphi_{k}$ are functions on $X$ which are l.s.c. near $\bar{x}_{1}, \ldots, \bar{x}_{k}$ respectively, and finite at these points. Suppose further that $\varphi$ is a $C^{2}$-function on $X \times \cdots \times X$ and the function $\left(x_{1}, \ldots, x_{k}\right) \mapsto$ $\varphi_{1}\left(x_{1}\right)+\cdots+\varphi_{k}\left(x_{k}\right)-\varphi\left(x_{1}, \ldots, x_{k}\right)$ attains a local minimum at $\bar{x}_{1}, \ldots, \bar{x}_{k}$. Let $H=D^{2} \varphi\left(\bar{x}_{1}, \ldots, \bar{x}_{k}\right)$. Then for any $\varepsilon>0$ there are $\left(x_{i}^{*}, A_{i}\right) \in \bar{\partial}^{2} \varphi_{i}\left(\bar{x}_{i}\right)$ such that 
$\left(x_{1}^{*}, \ldots, x_{k}^{*}\right)=\nabla \varphi\left(\bar{x}_{1}, \ldots, \bar{x}_{k}\right)$ and

$$
\left(\begin{array}{cccc}
A_{1} & 0 & \cdots & 0 \\
0 & A_{2} & 0 & \vdots \\
\vdots & 0 & \ddots & \vdots \\
0 & \cdots & \cdots & A_{k}
\end{array}\right) \geq H-\varepsilon I .
$$

If moreover $\varphi_{1}, \ldots, \varphi_{k}$ are convex, we may assume that $A_{i} \geq 0$.

Proof of Theorem 3.1. It is sufficient to consider the case of two functions, say $f$ and $g$, and assume that $x=0, x^{*}=0, A=0, f(x)=g(x)=0$.

So let $(0,0) \in \partial_{-}^{2}(f+g)(0)$. As both $f$ and $g$ are l.s.c., given an $\varepsilon>0$, we can find a $\rho>0$ so small that

$$
f(x) \geq-\epsilon, g(x) \geq-\epsilon \text { if }\|x\| \leq \rho .
$$

By Proposition 2.1 there is a $0<\delta<\min (\varepsilon, \rho)$ such that

$$
f(x)+g(x)+\frac{1}{2} \varepsilon\|x\|^{2}>0 \quad \text { if }\|x\| \leq \delta, x \neq 0 .
$$

Set

$$
h_{n}(u, v)=f(u)+g(v)+\frac{n}{2}\|u-v\|^{2}+\frac{\varepsilon}{2}\left(\|u\|^{2}+\|v\|^{2}\right) .
$$

We have $h_{n}(0,0)=0$, and $h_{n}(u, v) \geq-2 \varepsilon$ if $\|u\| \leq \delta,\|v\| \leq \delta$. Let $\left(u_{n}, v_{n}\right)$ be a point at which $h_{n}$ attains its minimum on $B(0, \delta) \times B(0, \delta)$. As $h_{n}\left(u_{n}, v_{n}\right) \leq$ $h_{n}(0,0)=0$, we conclude from $(3)$ that

$$
\frac{n}{2}\left\|u_{n}-v_{n}\right\|^{2} \leq 2 \varepsilon
$$

which means that $\left\|u_{n}-v_{n}\right\| \rightarrow 0$ as $n \rightarrow \infty$.

Assuming without loss of generality that $\left(u_{n}\right)$ and $\left(v_{n}\right)$ converge, we conclude that they have a common limit point $w$. Then, by lower semicontinuity,

$$
f(w)+g(w)+\varepsilon\|w\|^{2} \leq \liminf _{n \rightarrow \infty} h_{n}\left(u_{n}, v_{n}\right) \leq 0
$$

and (4) implies that $w=0$. Thus $\left(u_{n}\right) \rightarrow 0$ and $\left(v_{n}\right) \rightarrow 0$; in particular $\left\|u_{n}\right\|<$ $\min \left(\frac{\varepsilon}{2}, \frac{1}{2}\right)$ and $\left\|v_{n}\right\|<\min \left(\frac{\varepsilon}{2}, \frac{1}{2}\right)$ for large $n$. From now on we fix such an $n$.

We are now in a situation suitable for application of Lemma 3.2 (with $\varphi_{1}=f$, $\varphi_{2}=g$ and $\left.\varphi(u, v)=-\frac{1}{2}\left(n\|u-v\|^{2}+\varepsilon\|u\|^{2}+\varepsilon\|v\|^{2}\right)\right)$. Observe that as $\varphi$ is a quadratic function, it coincides with the quadratic function associated with its Hessian at 0 , and

$$
\nabla \varphi\left(u_{n}, v_{n}\right)=\left(-n\left(u_{n}-v_{n}\right)-\varepsilon u_{n}, n\left(u_{n}-v_{n}\right)-\varepsilon v_{n}\right) .
$$

Thus, applying Lemma 3.2 along with the definition of limiting 2-subjets, we find points $y$ and $z$ within $\varepsilon / 2$ of $u_{n}, v_{n}$, hence within $\varepsilon$ of 0 , such that $|f(y)|<\varepsilon / 2$, $|g(z)|<\varepsilon / 2$ and $\left(y^{*}, A\right) \in \partial_{-}^{2} f(y),\left(z^{*}, B\right) \in \partial_{-}^{2} g(z)$, with

$$
y^{*}=-n\left(u_{n}-v_{n}\right)-\varepsilon u_{n}, \quad z^{*}=n\left(u_{n}-v_{n}\right)+\varepsilon v_{n}
$$

and

$$
(A u \mid u)+(B v \mid v) \geq \varphi(u, v)-\frac{\varepsilon}{2}\left(\|u\|^{2}+\|v\|^{2}\right) \quad \forall u, v .
$$

From (5) we get

$$
\left\|y^{*}+z^{*}\right\| \leq \varepsilon\left(\left\|u_{n}\right\|+\left\|v_{n}\right\|\right)<\varepsilon
$$


and from (6), setting $u=v$,

$$
(A u \mid u)+(B u \mid u) \geq-2 \varepsilon\|u\|^{2}, \quad \forall u,
$$

that is, that $A+B \geq-2 \varepsilon I$. This completes the proof of the first part of the theorem. To prove the second statement we only need to observe that by Lemma 3.2 both $A$ and $B$ can be chosen positive semidefinite if both functions are convex.

Next we consider the composition operation, first for the simple case when the interior map is a projection.

Proposition 3.3. Let $x \in X, y \in Y$, and let $g: Y \rightarrow \mathbb{R} \cup\{\infty\}$ be l.s.c. near $y$ and finite at $y$. Consider the function $f$ on $X \times Y$ given by $f(u, v)=g(v)$.

(a) For any $\left(y^{*}, B\right) \in \partial_{-}^{2} g(y)$ and any $A \in L_{s}(X), A \leq 0, S \in L(X, Y)$ one has, setting $D:=B+S \circ A \circ S^{*}$ and $C:=S \circ A$,

$$
\left(\left(0, y^{*}\right), Q(A, D, C)\right) \in \partial_{-}^{2} f(x, y)
$$

in particular, $\left(\left(0, y^{*}\right), Q(0, B, 0)\right) \in \partial_{-}^{2} f(x, y)$.

(b) Conversely, if $\left(\left(x^{*}, y^{*}\right), P\right) \in \partial_{-}^{2} f(x, y)$ and $P=Q(A, D, C)$, then $x^{*}=0$, $A \leq 0$ and for any positive $\alpha$ there exists an $S \in L(X, Y)$ such that $C=S \circ(A-\alpha I)$, $B:=D-S \circ(A-\alpha I) \circ S^{*} \in \partial_{-}^{2} g\left(y, y^{*}\right)$ and $Q(0, B, 0) \geq P-Q(\alpha I, 0,0)$.

Proof. (a) The inclusion $\left(\left(0, y^{*}\right), Q(0, B, 0)\right) \in \partial_{-}^{2} f(x, y)$ for any $x$ is trivial. On the other hand, for $D=B+S \circ A \circ S^{*}, C=S \circ A$ we have

$$
\begin{gathered}
((Q(A, D, C)-Q(0, B, 0))(u, v) \mid(u, v)) \\
\quad=(A u \mid u)+2(S A u \mid v)+\left(S A S^{*} v \mid v\right) \\
\quad=\left(A\left(u+S^{*} v\right) \mid u+S^{*} v\right) \leq 0 .
\end{gathered}
$$

(b) By Proposition 2.1, for any $\varepsilon>0$ there exists $\rho>0$ such that

$$
\begin{aligned}
& f(x+u, y+v)-f(x, y)-\left(x^{*} \mid u\right)-\left(y^{*} \mid v\right) \\
& \quad \geq \frac{1}{2}((A u \mid u)+2(C u \mid v)+(D v \mid v))-\varepsilon\left(\|u\|^{2}+\|v\|^{2}\right)
\end{aligned}
$$

whenever $\|(u, v)\| \leq \rho$. Taking $v=0$ here, we get from (7)

$$
\left(x^{*} \mid u\right) \leq-\frac{1}{2}(A u \mid u)+\varepsilon\|u\|^{2} \text { when }\|u\| \leq \rho,
$$

which implies that $x^{*}=0$, and $A \leq 2 \varepsilon I$. As this is true for any $\varepsilon$, we conclude that $A \leq 0$. Therefore, for any $\alpha>0$, the operator $A_{\alpha}=A-\alpha I$ is nonsingular; set $S=C \circ A_{\alpha}^{-1}$.

From (7) we have

$$
g(y+v)-g(y)-\left(y^{*} \mid v\right) \geq \frac{1}{2}\left(\left(A_{\alpha} u \mid u\right)+2(C u \mid v)+(D v \mid v)\right)-\varepsilon\left(\|u\|^{2}+\|v\|^{2}\right)
$$

for all $(u, v)$ close to zero. Taking $u=-S^{*} v$ in this inequality with $\|v\|$ small enough, and setting $\varepsilon^{\prime}:=\varepsilon\left(1+\left\|S^{*}\right\|\right)$ we get

$$
\begin{aligned}
g(y+ & v)-g(y)-\left(y^{*} \mid v\right) \\
& \geq \frac{1}{2}\left(S A_{\alpha} S^{*} v \mid v\right)-\left(C S^{*} v \mid v\right)+\frac{1}{2}(D v \mid v)-\varepsilon^{\prime}\|v\|^{2} \\
& =\frac{1}{2}\left(\left(D-S A_{\alpha} S^{*}\right) v \mid v\right)-\varepsilon\left(1+\left\|S^{*}\right\|\right)\|v\|^{2} .
\end{aligned}
$$

As $\varepsilon$ is an arbitrary positive number, we conclude that $B:=D-S A_{\alpha} S^{*} \in$ $\partial_{-}^{2} g\left(y, y^{*}\right)$. The last inequality of the statement follows from part (a), in which we have shown that $Q\left(A_{\alpha}, D, C\right) \leq Q(0, B, 0)$ when $D=B+S A_{\alpha} S^{*}$ with $A_{\alpha} \leq 0$. 
Using the proposition, we can establish the following general fuzzy chain rule. It can also be written in an equality form which closely emulates the smooth case.

Theorem 3.4. Let $F: X \rightarrow Y$ be a map of class $C^{1}$ near $x \in X$ and let the function $g$ on $Y$ be l.s.c. near $y=F(x)$ and finite at $y$. Set $f=g \circ F$, and assume that $\left(x^{*}, A\right) \in \partial_{-}^{2} f(x)$. Then, for any $\varepsilon>0$, there are $u, u^{*} \in X, v, v^{*}, w^{*} \in Y$, $B \in L_{s}(Y)$ and $C \in L_{s}(X)$ such that $\|u-x\|<\varepsilon,\|v-y\|<\varepsilon,\left\|u^{*}-x^{*}\right\|<\varepsilon$, $\left\|v^{*}-w^{*}\right\|<\varepsilon$, and

$$
\begin{gathered}
\left(u^{*}, C\right) \in \partial_{-}^{2}\left(w^{*} \circ F\right)(u), \quad\left(v^{*}, B\right) \in \partial_{-}^{2} g(v), \\
C+F^{\prime}(u)^{*} \circ B \circ F^{\prime}(u) \geq A-\varepsilon I .
\end{gathered}
$$

Proof. As in the proof of Theorem 3.1, we can assume for simplicity that $x=0$, $y=0$. Fix an $\varepsilon>0$ so small that $F$ is $C^{1}$ in the $2 \varepsilon$-ball around zero, and let $k \geq\left\|F^{\prime}(u)\right\|$ for all $u$ with $\|u\| \leq \varepsilon$.

Set $h_{1}(u, v)=g(v)$, denote by $h_{2}$ the indicator function of the graph of $F$, and let $h=h_{1}+h_{2}$. Then for any $(u, v)$ either $h(u, v)=\infty \geq f(u)$, or $v=F(u)$ and $h(u, v)=g(v)=f(u)$. Therefore for any $\delta>0$

$$
h(u, v)-h(0,0) \geq f(u)-f(0) \geq\left(x^{*} \mid u\right)+\frac{1}{2}\left[(A u \mid u)-\delta\|u\|^{2}\right],
$$

provided $u$ is sufficiently close to zero. This means that

$$
\left(\left(x^{*}, 0\right), Q(A, 0,0)\right) \in \partial_{-}^{2} h(0,0) .
$$

Applying Theorem 3.1, we can find $x_{i}, y_{i}, x_{i}^{*}, y_{i}^{*}$ and $P_{i}, i=1,2$, such that

$$
\begin{gathered}
\left(\left(x_{i}^{*}, y_{i}^{*}\right), P_{i}\right) \in \partial_{-}^{2} h_{i}\left(x_{i}, y_{i}\right), \quad i=1,2, \\
P_{1}+P_{2} \geq Q(A, 0,0)-\varepsilon\left(2+2 k^{2}\right)^{-1} I, \\
\left\|x_{1}^{*}+x_{2}^{*}-x^{*}\right\|<\varepsilon, \quad\left\|y_{1}^{*}+y_{2}^{*}\right\|<\varepsilon .
\end{gathered}
$$

By Proposition 3.3 we have $x_{1}^{*}=0$, and there is a $B \in L_{s}(Y)$ such that $\left(y_{1}^{*}, B\right) \in$ $\partial_{-}^{2} g\left(y_{1}\right)$ and $Q(0, B, 0) \geq P_{1}-Q\left(\varepsilon\left(2+2 k^{2}\right)^{-1} I, 0,0\right)$, so that

$$
Q(0, B, 0)+P_{2} \geq Q(A, 0,0)-\varepsilon\left(1+k^{2}\right)^{-1} I .
$$

On the other hand, if we set

$$
G(u)=F\left(x_{2}+u\right)-F\left(x_{2}\right),
$$

then the inclusion for $i=2$ in (10) implies in particular that for any $\delta>0$

$$
0 \geq\left(x_{2}^{*} \mid u\right)+\left(y_{2}^{*} \mid G(u)\right)+\frac{1}{2}\left(P_{2}(u, G(u)) \mid(u, G(u))\right)-\delta\left(\|u\|^{2}+\|G(u)\|^{2}\right)
$$

for all sufficiently small $u$.

As $F$ is differentiable at $x_{2}$, we have

$$
G(u)=F^{\prime}\left(x_{2}\right) u+o(\|u\|) .
$$

Furthermore, defining $C \in L_{s}(X)$ by

$$
(C u \mid u)=\left(P_{2}\left(u, F^{\prime}\left(x_{2}\right) u\right) \mid\left(u, F^{\prime}\left(x_{2}\right) u\right)\right)
$$

and calculating the values of the corresponding quadratic forms at $\left(u, F^{\prime}\left(x_{2}\right) u\right)$, we find from (11) that

$$
F^{\prime *}\left(x_{2}\right) \circ B \circ F^{\prime}\left(x_{2}\right)+C \geq A-\varepsilon I .
$$

On the other hand, we obtain from (12), (13) that for any $\delta>0$ 


$$
-\left(y_{2}^{*} \mid F\left(x_{2}+u\right)-F\left(x_{2}\right)\right) \geq\left(x_{2}^{*} \mid u\right)+\frac{1}{2}(C u \mid u)-\delta\|u\|^{2},
$$

provided $u$ is sufficiently close to zero.

By definition this means that

$$
\left(x_{2}^{*}, C\right) \in \partial_{-}^{2}\left(-y_{2}^{*} \circ F\right)\left(x_{2}\right) .
$$

To conclude the proof, it remains to set $v^{*}=y_{1}^{*}, w^{*}=-y_{2}^{*}, u=x_{2}, u^{*}=x_{2}^{*}$.

Let us observe that it can be shown that Theorem 3.1 is in turn a consequence of Theorem 3.4. It suffices to take $Y=X^{k}, F(x)=(x, \ldots, x), g\left(y_{1}, \ldots, y_{k}\right)=$ $f_{1}\left(y_{1}\right)+\cdots+f_{k}\left(y_{k}\right)$ in the following corollary.

Corollary 3.5. Suppose $F: X \rightarrow Y$ is twice differentiable around $x$ (resp. linear and continuous) and $g: Y \rightarrow \mathbb{R} \cup\{\infty\}$ is l.s.c. around $y=F(x)$ and finite at $y$. Let $f=g \circ F$. Then for any $\left(x^{*}, A\right) \in \partial_{-}^{2} f(x)$ and any $\varepsilon>0$ there are $u, u^{*} \in X$, $v, v^{*}, w^{*} \in Y, B \in L_{s}(Y)$ such that $\|u-x\|<\varepsilon,\|v-y\|<\varepsilon,\left\|u^{*}-x^{*}\right\|<\varepsilon$, $\left\|v^{*}-w^{*}\right\|<\varepsilon,|g(v)-g(y)|<\varepsilon, u^{*}=w^{*} \circ F^{\prime}(u),\left(v^{*}, B\right) \in \partial_{-}^{2} g(v)$, and

$$
w^{*} \circ F^{\prime \prime}(u)+F^{\prime}(u)^{*} \circ B \circ F^{\prime}(u) \geq A-\varepsilon I \quad\left(\text { resp. } F^{\prime}(u)^{*} \circ B \circ F^{\prime}(u) \geq A-\varepsilon I\right) .
$$

\section{EFFICIENT SUBHESSIANS AND SINGULAR SUBJETS}

The set $\partial_{-}^{2} f\left(x, x^{*}\right)$, if nonempty, is necessarily unbounded, since with any $A$ it contains $A+B$ if $B \leq 0$. This may be a source of trouble when calculating limits, if we try to pass from the fuzzy calculus of Fréchet 2-subjets to an "exact" calculus of limiting 2-subjets. In addition, $A+B$ can hardly provide more information about $f$ than $A$ itself if $A \in \partial_{-}^{2} f\left(x, x^{*}\right)$ and $B \leq 0$. It is therefore worth trying to get rid of these "parasite" elements.

Passing to maximal elements with respect to the usual order $\geq$ on $L_{s}(X)$ is of no help, for it may just happen that no such elements exist as, say, in case of $X=\mathbb{R}$, $f(x)=|x|, x=0, x^{*}=0$ (in this case $\partial_{-}^{2} f\left(x, x^{*}\right)=L_{s}(\mathbb{R})$ ). We use a different strategy, selecting elements of $\partial_{-}^{2} f\left(x, x^{*}\right)$ which are representative enough. The following definition gives a precise sense to this expression.

Definition 4.1. Given a subset $\mathcal{A}$ of $L_{s}(X)$ and an $A \in \mathcal{A}$, we say that $A$ is efficient in $\mathcal{A}$ if for each $B \in \mathcal{A}$ with $B \geq A$ we have $\|B\| \geq\|A\|$.

In other words, $A$ is efficient in $\mathcal{A}$ if

$$
\|A\|=\min \{\|B\|: B \geq A, B \in \mathcal{A}\} .
$$

This definition corresponds to the usual notion of efficiency (or maximality) in the sense of Pareto for the multiobjective criteria $A \mapsto(A,-\|A\|)$ when $L_{s}(X) \times \mathbb{R}$ is endowed with the strict preorder associated with the pointed cone $L_{s}^{+}(X) \times(0, \infty)$ : $A$ is efficient if and only if there is no $B \geq A$ with $-\|B\|>-\|A\|$.

Efficient elements of $\partial_{-}^{2} f\left(x, x^{*}\right)$ will be called efficient subhessians of $f$ at $x$ w.r.t. $x^{*}$. We shall denote the collection of such elements by $\partial_{\text {eff }}^{2} f\left(x, x^{*}\right)$, and we set

$$
\partial_{\text {eff }}^{2} f(x)=\left\{\left(x^{*}, A\right): A \in \partial_{\text {eff }}^{2} f\left(x, x^{*}\right)\right\} .
$$

The following existence result is an immediate consequence of the fact that any nonempty closed subset of $L_{s}(X)$ has an element of least norm. 
Proposition 4.1. If $\partial_{-}^{2} f\left(x, x^{*}\right)$ is nonempty then $\partial_{\mathrm{eff}}^{2} f\left(x, x^{*}\right)$ is also nonempty. Moreover, for any $C \in \partial_{-}^{2} f\left(x, x^{*}\right)$ there is an $A \in \partial_{\mathrm{eff}}^{2} f\left(x, x^{*}\right)$ such that $A \geq C$ and $\|A\| \leq\|C\|$.

Examples. (a) A positive semidefinite matrix $A$ is efficient in any subset $\mathcal{A}$ of $L_{s}(X)$ containing it, since the norm is nondecreasing on the cone of positive semidefinite matrices.

(b) If $L_{s}(X)$ is endowed with the supremum norm, a matrix $A$ such that $\|A\|=$ $\left\|A^{+}\right\|$is efficient in any subset $\mathcal{A}$ of $L_{s}(X)$ containing it: if $B \geq A$, the greatest eigenvalue of $B$ is not less than the greatest eigenvalue of $A$, so that $\|B\| \geq\|A\|$. Here $A^{+}=\frac{1}{2}(|A|+A)$ and $A^{-}=\frac{1}{2}\left(|A|-A\right.$ ), with $|A|=\left(A^{2}\right)^{1 / 2}$ (see [13] for instance).

(c) Let $\mathcal{A}$ be a subset of $L_{s}(X)$ which is stable by addition of elements of $L_{s}^{+}(X)$, or more generally, which is such that for any $A \in \mathcal{A}$ one has $A^{+} \in \mathcal{A}$. Suppose the norm on $L_{s}(X)$ satisfies

$$
\|A\| \geq \max \left(\left\|A^{+}\right\|,\left\|A^{-}\right\|\right)
$$

for each $A \in L_{s}(X)$. This assumption is satisfied when $\|\cdot\|$ is the supremum norm or the Frobenius norm. We claim that if $A$ is efficient in $\mathcal{A}$ then $\|A\|=\left\|A^{+}\right\|$. In fact, if $A$ is efficient, as $A^{+} \geq A$ we have $\left\|A^{+}\right\| \geq\|A\| \geq \max \left(\left\|A^{+}\right\|,\left\|A^{-}\right\|\right) \geq\left\|A^{+}\right\|$ and $\left\|A^{+}\right\|=\|A\|$. Moreover, if the norm is such that $\|A\|>\left\|A^{+}\right\|$when $A^{-} \neq 0$, and if $A$ is efficient in $\mathcal{A}$, then $A^{-}=0$ and $A \geq 0$. Let us observe that this property holds when the norm is such that

$$
\|A\|=\left(\left\|A^{+}\right\|^{2}+\left\|A^{-}\right\|^{2}\right)^{1 / 2}
$$

in particular for the Frobenius norm. Taking Example (a) into account (and the fact that if $A$ is efficient in $L_{s}(X)$ it is efficient in any subset), we get that for such a norm $A$ is efficient in $\mathcal{A}$ iff $A \geq 0$.

(d) Let us consider the set $\mathcal{A}:=\left\{A \in L_{s}(X): A \leq H\right\}$, where $H$ is a given element of $L_{s}(X)$ when this space is endowed with the supremum norm. We claim that if $A$ is not positive semidefinite then $A$ is efficient in $\mathcal{A}$, provided $A \geq \mu I$, where $\mu$ is the minimal eigenvalue of $H$. (If $A$ is positive semidefinite, it is efficient by (a).) For $B \in \mathcal{A}, B \geq A$, we have

$$
\mu \leq \min _{v \in S}(A v \mid v) \leq \min _{v \in S}(B v \mid v) \leq \min _{v \in S}(H v \mid v)=\mu
$$

so that equality holds in these relations, and $\max _{v \in S}(A v \mid v) \leq \max _{v \in S}(B v \mid v)$, so that we get $\|B\| \geq\|A\|$, which means that $A$ is efficient.

(e) Consider now two spaces $X, Y$ and a function $g$ on $Y$; let us define $f$ on $X \times Y$ by $f(x, y):=g(y)$. Suppose that for each $B \in L_{s}(Y)$ one has $\|Q(0, B, 0)\|=\|B\|$; this is the case with the usual norms considered above. Let us prove that for any $y^{*} \in \partial_{-} g(y)$ and any $B \in \partial_{\text {eff }}^{2} g\left(y, y^{*}\right)$ one has $Q(0, B, 0) \in \partial_{\text {eff }}^{2} f\left((x, y),\left(0, y^{*}\right)\right)$. We already know from Proposition 3.3 that $Q(0, B, 0) \in \partial^{2} f\left((x, y),\left(0, y^{*}\right)\right)$ and that for any $P:=Q(A, D, C) \in \partial^{2} f\left((x, y),\left(0, y^{*}\right)\right)$ we have $A \leq 0$. If $P \geq Q(0, B, 0)$, taking $v=0$ in the relation

$$
(A u \mid u)+2(C u \mid v)+(D v \mid v) \geq(B v \mid v), \quad \forall u, \forall v,
$$

we get $A \geq 0$ and $A=0$. Then, for each $v \in Y$, the linear form $u \mapsto(C u \mid v)$ is bounded below, hence is zero, and $C=0$. Thus $D \geq B$. Moreover, $D \in \partial^{2} g\left(y, y^{*}\right)$, as is easily seen. As $B$ is efficient in this set, we get $\|P\|=\|Q(0, D, 0)\|=\|D\| \geq$ $\|B\|=\|Q(0, B, 0)\|$, as expected. 
On the other hand, the example of the functions $f$ on $\mathbb{R}^{2}$ given by $f(x, y)=|y|$ shows that $\partial_{\text {eff }}^{2} f\left((x, y),\left(x^{*}, y^{*}\right)\right)$ may be larger than the set of $Q(0, B, 0)$ with $B \in$ $\partial_{\text {eff }}^{2} g\left(y, y^{*}\right)$, as for $(x, y)=(0,0),\left(x^{*}, y^{*}\right)=(0,0)$ the subhessian $Q(0,0, c)$ of $f$ at $((0,0),(0,0))$, with $c$ arbitrary, corresponding to the quadratic form $(u, v) \rightarrow c u v$, is efficient.

Definition 4.2. The set

$$
\partial_{\infty}^{2} f(x)=\limsup _{\substack{u f \\ \lambda \rightarrow 0}} \lambda \partial_{\text {eff }}^{2} f(u)
$$

will be called the singular 2-subjet of $f$ at $x$. If $F: X \rightarrow Y$ is continuous near $x$, then we define the singular 2-cosubjet of $F$ at $x$ by

$$
D_{\infty}^{2} F\left(y^{*}, x\right)=\limsup _{\substack{u \rightarrow x \\ z^{*} \rightarrow y^{*} \\ \lambda \rightarrow 0_{+}}} \lambda \partial_{\text {eff }}^{2}\left(z^{*} \circ F\right)(u) .
$$

Clearly, $\partial_{\infty}^{2} f(x)$ is a cone; it may, in principle, depend on the norm on $L_{s}(X)$. We note, however, that if $A$ and $A^{\prime}$ are singular 2-subjets of $f$ at $x$ corresponding to different norms $\|\cdot\|$ and $\|\cdot\|^{\prime}$ on $L_{s}(X)$, then for any $A \in \partial_{\infty}^{2} f(x)$ there is an $A^{\prime} \in \partial_{\infty}^{2} f(x)$ such that $A^{\prime} \geq A$ and $\left\|A^{\prime}\right\|^{\prime} \leq\|A\|^{\prime}$. This follows easily from the preceding proposition.

Let us examine singular subjets of some important classes of functions. Recall that $f$ is paraconvex [21], [18] (or semiconvex [5], or lower- $C^{2}$ [19]) at $x$ with index $\alpha \geq 0$ if there exists a convex neighborhood of $x$ on which $f+\frac{1}{2} \alpha\|\cdot\|^{2}$ is convex, finite and continuous; we call $f$ paraconcave at $x$ with index $\alpha$ if $-f$ is paraconvex with index $\alpha$. We say that $f$ is paraconvex (resp. paraconcave) on a subset $A$ of $X$ if it is paraconvex (resp. paraconcave) at each point of $A$.

Proposition 4.2. Suppose $f$ is paraconcave with index $\alpha$ on a ball $B(x, r)$ with center $x$ and radius $r$ and bounded above by $m$ on $B(x, r)$. Then for any $\rho \in(0, r)$ there exists $\beta$ in $\mathbb{R}_{+}$, depending only on $m, r, \rho$ and $\alpha$, such that for any $\left(u^{*}, A\right) \in$ $\partial_{-}^{2} f(u)$ with $u \in B(x, \rho)$ one has $\left\|u^{*}\right\| \leq \beta$ and $A \leq \alpha I$.

Therefore for any $u \in B(x, \rho),\left(u^{*}, A\right) \in \partial_{\infty}^{2} f(u)$ one has $u^{*}=0$ and $A \leq 0$.

Proof. We first observe that by [16], Theorem 5.1, the convex function $g$ given by $g(u)=-f(u)+\frac{1}{2} \alpha\|u-x\|^{2}$ is Lipschitzian on $B(x, \rho)$ with rate $\lambda:=2 m^{\prime}(\rho-r)^{-1}$, where $m^{\prime}=m+\frac{1}{2} \alpha \rho^{2}$. Thus $f$ is Lipschitzian with rate $\beta=\lambda+\alpha \rho$ on $B(x, \rho)$. It follows that for any $u \in \operatorname{int} B(x, \rho),\left(u^{*}, A\right) \in \partial_{-}^{2} f(u), v \in X$ we have

$$
\left\langle u^{*}, v\right\rangle \leq f^{\prime}(x, v) \leq \beta\|v\| ;
$$

hence $\left\|u^{*}\right\| \leq \beta$ and, for $v^{*}=-u^{*}+\alpha(u-x)$,

$$
(A v \mid v) \leq f_{-}^{\prime \prime}\left(u, u^{*}, v\right) \leq-g_{-}^{\prime \prime}\left(u, v^{*}, v\right)+\alpha\|v\|^{2} \leq \alpha\|v\|^{2},
$$

since $g_{-}^{\prime \prime}\left(u, v^{*}, v\right) \geq 0$ as $g$ is convex.

The last statement follows easily by taking limits.

A stronger conclusion can be given when $f$ is both paraconvex and paraconcave.

Proposition 4.3. If $f$ is both paraconvex and paraconcave near $x$, then there are $\beta>0, \mu>0$ and $\rho>0$ such that whenever $\|u-x\|<\rho$ and $\left(u^{*}, A\right) \in \partial_{-}^{2} f(u)$, one has $\left\|u^{*}\right\| \leq \beta$ and $\|A\| \leq \mu$. Therefore, in this case,

$$
\partial_{\infty}^{2} f(x)=\{(0,0)\}
$$


Proof. If the function is both paraconvex and paraconcave near $x$, then by a result of [6] it is of class $C^{1,1}$ (i.e. differentiable with a locally Lipschitzian derivative), and one can easily verify that if $\mu$ is a Lipschitz constant for $\nabla f$ in a neighborhood of $x$, then $\left|f_{-}^{\prime \prime}\left(u, u^{*}, v\right)\right| \leq \mu\|v\|^{2}$ for any $v \in X$ and $u$ in a neighborhood of $x$, with $u^{*}=\nabla f(u)$. Then Proposition 2.1 and Example (c) above give the result.

It should be observed that convex functions may have singular subhessians which are not positive semidefinite, as the following example shows.

Example. (f) Consider the convex function $f$ on $\mathbb{R}^{2}$ given by $f(x, y)=|x-y|$. Then the matrix

$$
A=\left(\begin{array}{cc}
n & 0 \\
0 & -n
\end{array}\right)=Q(n,-n, 0)
$$

clearly belongs to $\partial_{-}^{2} f(0,0)$. Let us show that $A \in \partial_{\text {eff }}^{2} f(0,0)$ if $L_{s}(X)$ is endowed either with the supremum norm or with the Frobenius norm. It is sufficient to show this only for $n=1$. The case of the supremum norm follows from Example (b) above. Consider the case of the Frobenius norm. Then, for $B=Q(\alpha, \beta, \gamma) \geq A=$ $Q(1,-1,0)$ and $B \in \partial_{-}^{2} f(0,0)$ we must have $\alpha \geq 1, \beta \geq-1, \gamma^{2}-(\alpha-1)(\beta+1) \leq 0$ and $\gamma=-\frac{1}{2}(\alpha+\beta)$. Then the minimization of $\|B\|^{2}=\alpha^{2}+\beta^{2}+2 \gamma^{2}$ under these constraints gives $\alpha=1, \beta=-1, \gamma=0$. It easily follows that $(0, Q(1,-1,0)) \in$ $\partial_{\infty}^{2} f(0)$.

\section{The MAIN RESUlts}

This section contains statements and proofs of the two main theorems about generalized 2-subjets of a sum of several functions and of a composition of a function and a map.

Theorem 5.1. Let $f_{1}, \ldots, f_{k}$ be extended real-valued functions on $X$ which are l.s.c. near $x$ and finite at $x$. Assume that the following qualification assumption holds:

(QA)

$$
\begin{gathered}
\text { if }\left(x_{i}^{*}, A_{i}\right) \in \partial_{\infty}^{2} f_{i}(x), i=1, \ldots, k \text { and } x_{1}^{*}+\cdots+x_{k}^{*}=0, A_{1}+\cdots+A_{k} \geq 0, \\
\text { then actually } x_{1}^{*}=\cdots=x_{k}^{*}=0, A_{1} \geq 0, \ldots, A_{k} \geq 0 .
\end{gathered}
$$

Then

$$
\bar{\partial}^{2}\left(f_{1}+\cdots+f_{k}\right)(x) \subset \bar{\partial}^{2} f_{1}(x)+\cdots+\bar{\partial}^{2} f_{k}(x) .
$$

The following two corollaries are easy consequences of the theorem.

Corollary 5.2. If all the functions $f_{1}, \ldots, f_{k}$ are paraconcave near $x$, then the conclusion of the theorem holds.

Proof. Apply Proposition 4.2.

Similarly, applying Proposition 4.3, one gets the following consequence.

Corollary 5.3. If the functions $f_{1}, \ldots, f_{k}$ are l.s.c. near $x$, finite at $x$ and all but at most one of them are both paraconvex and paraconcave near $x$, then the conclusion of the theorem holds.

As a particular case one gets: 
Corollary 5.4. If $f_{1}$ is l.s.c. near $x$ and finite at $x$, and if $f_{2}$ is $C^{2}$ near $x$, then

$$
\bar{\partial}^{2}\left(f_{1}+f_{2}\right)(x)=\bar{\partial}^{2} f_{1}(x)+\left(\nabla f_{2}(x), \nabla^{2} f_{2}(x)\right) .
$$

Proof. As $f_{2}$ is $C^{2}$, hence $C^{1,1}$, the inclusion

$$
\bar{\partial}^{2}\left(f_{1}+f_{2}\right)(x) \subset \bar{\partial}^{2} f_{1}(x)+\left(\nabla f_{2}(x), \nabla^{2} f_{2}(x)\right)
$$

follows from the preceding corollary. Setting $g_{1}=f_{1}+f_{2}$ and $g_{2}=-f_{2}$, we get the opposite conclusion.

Proof of Theorem 5.1. Let $f=f_{1}+\cdots+f_{k}$, and let $\left(x^{*}, A\right) \in \bar{\partial}^{2} f(x)$. This means that there exist sequences $\left(x_{n}\right),\left(x_{n}^{*}\right),\left(A_{n}\right)$ such that $\left(x_{n}\right) \rightarrow x,\left(f\left(x_{n}\right)\right) \rightarrow f(x)$, $\left(x_{n}^{*}\right) \rightarrow x^{*},\left(A_{n}\right) \rightarrow A$ and $\left(x_{n}^{*}, A_{n}\right) \in \partial_{-}^{2} f\left(x_{n}\right)$ for each $n \in \mathbb{N}$.

By Theorem 3.1, given a sequence $\left(\varepsilon_{n}\right)$ of positive numbers with limit 0 , there are sequences $\left(x_{i, n}\right),\left(x_{i, n}^{*}\right),\left(A_{i, n}\right)$ for $i=1, \ldots, k$ such that for each $i$ and each $n$ one has $\left\|x_{i, n}-x_{n}\right\| \leq \varepsilon_{n},\left|f_{i}\left(x_{i, n}\right)-f_{i}\left(x_{n}\right)\right| \leq \varepsilon_{n},\left\|\sum_{i=1}^{k} x_{i, n}^{*}-x_{n}^{*}\right\| \leq \varepsilon_{n}$ and

$$
\sum_{i=1}^{k} A_{i, n} \geq A_{n}-\varepsilon_{n} I, \quad\left(x_{i, n}^{*}, A_{i, n}\right) \in \partial_{-}^{2} f_{i}\left(x_{i, n}\right) .
$$

Replacing, if necessary, $A_{i, n}$ by other elements of $\partial_{-}^{2} f_{i}\left(x_{i}, x_{i, n}^{*}\right)$, we can make sure that $\sum_{i=1}^{k}\left\|A_{i, n}\right\|=\mu_{n}$ where

$$
\mu_{n}:=\min \left\{\sum_{i=1}^{k}\left\|B_{i}\right\|: B_{i} \in \partial_{-}^{2} f_{i}\left(x_{i, n}, x_{i, n}^{*}\right), \sum_{i=1}^{k} B_{i} \geq A_{n}-\varepsilon_{n} I\right\} .
$$

In this case $A_{i, n} \in \partial_{\text {eff }}^{2} f_{i}\left(x_{i, n}, x_{i, n}^{*}\right)$ for $i=1, \ldots, k, n \in \mathbb{N}$.

Let us set

$$
r_{n}=\sum_{i=1}^{k}\left(\left\|x_{i, n}^{*}\right\|+\left\|A_{i, n}\right\|\right) .
$$

When $\left(r_{n}\right)$ has a bounded subsequence we can extract a converging sequence of $\left(x_{i, n}^{*}, A_{1, n}, \ldots, x_{k, n}^{*}, A_{k, n}\right)$, and a passage to the limit in (14) concludes the proof.

So it remains to show that if $\left(r_{n}\right) \rightarrow \infty$ then we reach a contradiction. Let us set $u_{i, n}^{*}=r_{n}^{-1} x_{i, n}^{*}$ and $B_{i, n}=r_{n}^{-1} A_{i, n}$. Since the sequences $\left(u_{i, n}^{*}\right)_{n \geq 0}$ and $\left(B_{i, n}\right)_{n \geq 0}$ are bounded, we may assume that they converge to some $u_{i}^{*}$ and $B_{i}$ respectively, for $i=1, \ldots, k$. By definition, $\left(u_{i}^{*}, B_{i}\right) \in \partial_{\infty}^{2} f_{i}(x)$, and it follows from (14) that

$$
u_{1}^{*}+\cdots+u_{k}^{*}=0, \quad B_{1}+\cdots+B_{k} \geq 0,
$$

so that by $(\mathrm{QA})$ we get $u_{1}^{*}=0, \ldots, u_{k}^{*}=0$ and $B_{1} \geq 0, \ldots, B_{k} \geq 0$. Let $\beta_{i}=\left\|B_{i}\right\|$, so that $\beta_{1}+\cdots+\beta_{k}=1$, and set

$$
\begin{gathered}
D_{n}:=\sum_{i=1}^{k} A_{i, n}-\left(A_{n}-\varepsilon_{n} I\right) \geq 0, \\
A_{i, n}^{\prime}=A_{i, n}-\beta_{i} D_{n}=\left(1-\beta_{i}\right) A_{i, n}+\beta_{i}\left(A_{i, n}-D_{n}\right), \quad i=1, \ldots, k .
\end{gathered}
$$

Since $A_{i, n}^{\prime} \in \partial_{-}^{2} f_{i}\left(x_{i, n}, x_{i, n}^{*}\right)$ and

$$
\sum_{i=1}^{k} A_{i, n}^{\prime} \geq A_{n}-\varepsilon_{n} I
$$

we will reach the desired contradiction if we show that $\sum_{i=1}^{k}\left\|A_{i, n}^{\prime}\right\|<\mu_{n}$. 
Let us set

$$
\alpha_{n}=\left\|r_{n}^{-1}\left(A_{n}-\varepsilon_{n} I\right)\right\|+\sum_{i=1}^{k}\left\|r_{n}^{-1} A_{i, n}-B_{i}\right\|,
$$

so that $\left(\alpha_{n}\right) \rightarrow 0$. We have

$$
A_{i, n}-D_{n}=A_{n}-\varepsilon_{n} I-\sum_{j \neq i} A_{j, n}
$$

and for each $u$ in the unit sphere $S$ of $X$, as $B_{j} \geq 0$ for $j=1, \ldots, k$, we have

$$
\left(r_{n}^{-1}\left(A_{i, n}-D_{n}\right) u \mid u\right) \leq\left(r_{n}^{-1}\left(A_{n}-\varepsilon_{n} I\right) u \mid u\right)+\sum_{j \neq i}\left(\left(B_{j}-r_{n}^{-1} A_{j, n}\right) u \mid u\right) \leq \alpha_{n},
$$

as we may suppose without loss of generality that $|(T u \mid u)| \leq\|T\|$. Hence

$$
\begin{aligned}
\left(r_{n}^{-1} A_{i, n}^{\prime} u \mid u\right) & =\left(1-\beta_{i}\right)\left(r_{n}^{-1} A_{i, n} u \mid u\right)+\beta_{i}\left(r_{n}^{-1}\left(A_{i, n}-D_{n}\right) u \mid u\right) \\
& \leq\left(1-\beta_{i}\right)\left(B_{i} u \mid u\right)+\left(1-\beta_{i}\right) \alpha_{n}+\beta_{i} \alpha_{n} \\
& \leq\left(1-\beta_{i}\right) \beta_{i}+\alpha_{n}
\end{aligned}
$$

and similarly

$$
\begin{aligned}
\left(r_{n}^{-1} A_{i, n}^{\prime} u \mid u\right) & =\left(1-\beta_{i}\right)\left(r_{n}^{-1} A_{i, n} u \mid u\right)+\beta_{i}\left(r_{n}^{-1}\left(A_{n}-\varepsilon_{n} I\right) u \mid u\right)-\beta_{i} \sum_{j \neq i}\left(r_{n}^{-1} A_{j, n} u \mid u\right) \\
& \geq\left(1-\beta_{i}\right)\left(B_{i} u \mid u\right)-\beta_{i} \sum_{j \neq i}\left(B_{j} u \mid u\right)-\alpha_{n} \\
& \geq-\beta_{i} \sum_{j \neq i} \beta_{j}-\alpha_{n}=-\beta_{i}\left(1-\beta_{i}\right)-\alpha_{n}
\end{aligned}
$$

(again because $B_{i} \geq 0$ ). It follows that

$$
\left\|r_{n}^{-1} A_{i, n}^{\prime}\right\| \leq\left(1-\beta_{i}\right) \beta_{i}+\alpha_{n} .
$$

Since $\mu_{n}=\sum_{i=1}^{k}\left\|A_{i, n}\right\| \sim r_{n}$, we get

$$
\lim \sup \mu_{n}^{-1}\left(\sum_{i=1}^{k}\left\|A_{i, n}^{\prime}\right\|\right) \leq \sum_{i=1}^{k}\left(1-\beta_{i}\right) \beta_{i}<1
$$

and we arrive at the expected contradiction: $\sum_{i=1}^{k}\left\|A_{i, n}^{\prime}\right\|<\mu_{n}$ for $n$ large enough.

Now let us turn to composition.

Theorem 5.5. Let $f=g \circ F$, where $F: X \rightarrow Y$ is a map of class $C^{1}$ at $x$ and $g$ is a function on $Y$ which is l.s.c. and finite at $y:=F(x)$. Suppose the following condition is satisfied:

$$
\begin{aligned}
& \left(y^{*}, B^{*}\right) \in \partial_{\infty}^{2} g(y), \quad(0, C) \in D_{\infty}^{2} F\left(y^{*}, x\right), \\
& F^{\prime}(x)^{*} \circ B \circ F^{\prime}(x)+C \geq 0 \Rightarrow y^{*}=0, B=0 .
\end{aligned}
$$

Then for any $\left(x^{*}, A\right) \in \bar{\partial}^{2} f(x)$ there exist $\left(y^{*}, B\right) \in \bar{\partial}^{2} g(y)$ and $C \in L_{s}(X)$ such that $\left(x^{*}, C\right) \in \bar{D}^{2} F\left(y^{*}, x\right)$ and

$$
A=F^{\prime}(x)^{*} \circ B \circ F^{\prime}(x)+C .
$$


Proof. The proof is similar in structure to the proof of the previous theorem. Since calculus rules for efficient subhessians are not available, we cannot deduce the result for composition from the theorem for sums, as in the fuzzy case of Theorem 3.4. By definition there exists a sequence $\left(\left(x_{n}, x_{n}^{*}, A_{n}\right)\right)$ with limit $\left(x, x^{*}, A\right)$ such that $\left(f\left(x_{n}\right)\right) \rightarrow f(x)$ and $\left(x_{n}^{*}, A_{n}\right) \in \partial_{-}^{2} f\left(x_{n}\right)$ for each $n$. Let $\left(\varepsilon_{n}\right)$ be a sequence of positive numbers converging to 0 . Theorem 3.4 ensures that there are $u_{n}, u_{n}^{*} \in X$ and $v_{n}, v_{n}^{*}, y_{n}^{*} \in Y$ such that $\left\|x_{n}-u_{n}\right\|<\varepsilon_{n},\left\|v_{n}-y_{n}\right\|<\varepsilon_{n}$ for $y_{n}=F\left(x_{n}\right)$, $\left|g\left(v_{n}\right)-g\left(y_{n}\right)\right|<\varepsilon_{n},\left\|x_{n}^{*}-u_{n}^{*}\right\|<\varepsilon_{n}$, and $\left\|v_{n}^{*}-y_{n}^{*}\right\|<\varepsilon_{n}$, and such that the set $\mathcal{M}_{n}$ of $(B, C) \in L_{s}(X) \times L_{s}(Y)$ satisfying

$$
\begin{gathered}
\left(v_{n}^{*}, B\right) \in \partial_{-}^{2} g\left(v_{n}\right), \quad\left(u_{n}^{*}, C\right) \in \partial_{-}^{2}\left(y_{n}^{*} \circ F\right)\left(u_{n}\right), \\
u_{n}^{*}=F^{\prime}\left(u_{n}\right)^{*} y_{n}^{*}, \quad F^{\prime}\left(u_{n}\right)^{*} \circ B \circ F^{\prime}\left(u_{n}\right)+C \geq A_{n}-\varepsilon_{n} I
\end{gathered}
$$

is nonempty. We choose $\left(B_{n}, C_{n}\right)$ in this set in such a way that

$$
\left\|B_{n}\right\|+\left\|C_{n}\right\|=\min \left\{\|B\|+\|C\|:(B, C) \in \mathcal{M}_{n}\right\} .
$$

This is possible since $\mathcal{M}_{n}$ is closed. Let us set

$$
r_{n}=\left\|y_{n}^{*}\right\|+\left\|B_{n}\right\|+\left\|C_{n}\right\| .
$$

When $\left(r_{n}\right)$ has a bounded subsequence we conclude the proof by passing to a converging subsequence of $\left(\left(y_{n}^{*}, B_{n}, C_{n}\right)\right)$, replacing the limit point $C$ of $\left(C_{n}\right)$ by some $C^{\prime} \leq C$ if necessary.

It remains to prove that $\left(r_{n}\right) \rightarrow \infty$ leads to a contradiction with condition (QC). Let us set

$$
y^{*}=\lim r_{n}^{-1} y_{n}^{*}, \quad B=\lim r_{n}^{-1} B_{n}, \quad C=\lim r_{n}^{-1} C_{n},
$$

assuming without loss of generality that the limits exist. Since our choice of $\left(B_{n}, C_{n}\right)$ in $\mathcal{M}_{n}$ ensures that $B_{n} \in \partial_{\text {eff }}^{2} g\left(v_{n}, v_{n}^{*}\right)$ and $C_{n} \in \partial_{\text {eff }}^{2}\left(y_{n}^{*} \circ F\right)\left(u_{n}, u_{n}^{*}\right)$, we get

$$
\left(y^{*}, B\right) \in \partial_{\infty}^{2} g(y), \quad(0, C) \in \partial_{\infty}^{2} F\left(y^{*}, x\right), \quad F^{\prime}(x)^{*} \circ B \circ F^{\prime}(x)+C \geq 0 .
$$

It follows from (QC) that $y^{*}=0, B=0, C \geq 0$. Thus $\left\|B_{n}\right\|+\left\|C_{n}\right\| \sim\left\|C_{n}\right\| \sim r_{n}$. Setting

$$
C_{n}^{\prime}=A_{n}-\varepsilon_{n} I-F^{\prime}\left(u_{n}\right)^{*} \circ B_{n} \circ F^{\prime}\left(u_{n}\right),
$$

we see that $C_{n}^{\prime} \leq C_{n},\left(B_{n}, C_{n}^{\prime}\right) \in \mathcal{M}_{n}$ and

$$
\lim \left\|C_{n}\right\|^{-1}\left\|C_{n}^{\prime}\right\|=\lim \left\|r_{n}^{-1}\left(A_{n}-\varepsilon_{n} I\right)-F^{\prime}\left(u_{n}\right)^{*} \circ r_{n}^{-1} B_{n} \circ F^{\prime}\left(u_{n}\right)\right\|=0,
$$

so that $\left\|B_{n}\right\|+\left\|C_{n}^{\prime}\right\|<\left\|B_{n}\right\|+\left\|C_{n}\right\|$ for $n$ large enough, a contradiction with (17).

Applying the proof of Theorem 5.5 (with a reference to Corollary 3.5 rather than to Theorem 3.1) to the case of twice continuously differentiable $F$, we get the following result.

Corollary 5.6. Suppose that $F: X \rightarrow Y$ is twice continuously differentiable near $x$, and let $g$ be a l.s.c. function on $Y$ which is finite at $y=F(x)$. Set $f=g \circ F$ and assume that the following qualification condition is satisfied:

$$
\left(y^{*}, B\right) \in \partial_{\infty}^{2} g(y), F^{\prime}(x)^{*} \circ B \circ F^{\prime}(x)+y^{*} \circ F^{\prime \prime}(x) \geq 0 \Rightarrow y^{*}=0, B=0 .
$$

Then for any $\left(x^{*}, A\right) \in \bar{\partial}^{2} f(x)$ there is a $\left(y^{*}, B\right) \in \bar{\partial}^{2} g(y)$ such that

$$
A \leq F^{\prime}(x)^{*} \circ B \circ F^{\prime}(x)+y^{*} \circ F^{\prime \prime}(x) .
$$


In particular, when $F$ is a linear mapping we get

Corollary 5.7. If $F: X \rightarrow Y$ is a linear mapping, $f=g \circ F$ and

$$
\left(y^{*}, B\right) \in \partial_{\infty}^{2} g(y), F^{*} \circ B \circ F \geq 0 \Rightarrow y^{*}=0, B=0
$$

then for any $\left(x^{*}, A\right) \in \bar{\partial}^{2} f(x)$ there is a $\left(y^{*}, B\right) \in \bar{\partial}^{2} g(y)$ such that $A \leq F^{*} \circ B \circ F$.

For the still more special case of $F$ a projection operator, Proposition 3.3 allows us to obtain a slightly weaker form of second order chain rule but without any qualification condition.

Proposition 5.8. Let $g$ be an l.s.c. function on $Y$, let $X$ be another space, and let the function $f$ on $X \times Y$ be defined by $f(x, y)=g(y)$.

(a) For any $\left(y^{*}, B\right) \in \bar{\partial}^{2} g(y), A \in L_{s}(X), A \leq 0, x \in X$ and $S \in L(X, Y)$,

$$
\left(\left(0, y^{*}\right), Q(A, D, C)\right) \in \bar{\partial}^{2} f(x, y)
$$

where $D=B+S A S^{*}, C=S A$.

(b) Conversely, if $\left(\left(x^{*}, y^{*}\right), Q(A, D, C)\right) \in \bar{\partial}^{2} f(x, y)$, then $x^{*}=0, A \leq 0$, and, for any $\alpha>0$,

$$
\left(y^{*}, B\right) \in \bar{\partial}^{2} g(y), \quad Q(0, B, 0) \geq Q(A, D, C)-\alpha I,
$$

where $S=C(A-\alpha I)^{-1}, B=D-S(A-\alpha I)^{-1} S^{*}$.

Proof. Both parts follow directly from the corresponding parts of Proposition 3.3 when we apply the definition of the limiting subjet and pass to the limit. It only has to be observed in the proof of the second part that the supremum norm of $(A-\alpha I)^{-1}$ is not greater than $\alpha^{-1}$, so whenever $\left(\left(0, y_{n}^{*}\right), Q\left(A_{n}, D_{n}, C_{n}\right)\right) \in \partial_{-}^{2} f\left(x, y_{n}\right)$ and $A_{n}, D_{n}, C_{n}$ converge to certain $A, D, C$, then the operators $S_{n}=C_{n}\left(A_{n}-\alpha I\right)^{-1}$ are uniformly bounded, and so are the operators $B_{n}=D_{n}-S_{n}\left(A_{n}-\alpha I\right)^{-1} S_{n}^{*}$.

We conclude this section with the observation that, contrary to the fuzzy calculus rules of Theorems 3.1 and 3.4, we cannot state that Theorems 5.1 and 5.5 are equivalent. Theorem 5.1 does not follow from Corollary 5.7, for the qualification condition of the latter, $B=0$, would result in the stronger requirement that $A_{1}=$ $\cdots=A_{n}=0$ in the situation of Theorem 5.1. We must note, however, that Corollary 5.2 does follow from Corollary 5.6 in that way.

On the other hand, we could use Theorem 5.1 to get a chain rule for a composed function using the same approach as in the proof of Theorem 3.4. But this would result in a different (from Theorem 5.5) statement involving efficient subhessians of the function $f(x, y)=g(y)$ of two variables in the qualification condition, which, as we have seen in Example (e) of the preceding section, is substantially bigger than what would come from the set of efficient subhessians of $g$.

\section{Comparison With other Works}

Let us first clarify how the notion of approximate subhessian is related to the concept of generalized hessian. Recall that for $f: X \rightarrow \mathbb{R}^{\bullet}:=\mathbb{R} \cup\{\infty\}$ the generalized hessian $\bar{\nabla}^{2} f\left(x, x^{*}\right)$ of $f$ at $\left(x, x^{*}\right) \in X \times X$ is the set of limits of sequences $\left(\nabla^{2} f\left(x_{n}\right)\right)$, where $\left(x_{n}\right)$ is a sequence in the domain of the second order derivative $\nabla^{2} f$ of $f$ such that $\left(x_{n}\right) \rightarrow x,\left(f\left(x_{n}\right)\right) \rightarrow f(x),\left(\nabla f\left(x_{n}\right)\right) \rightarrow x^{*}$. This notion has mainly been considered in the case when $f$ is of class $C^{1,1}$. 
Proposition 6.1. For any l.s.c. $f: X \rightarrow \mathbb{R}^{\bullet}$ and $\left(x, x^{*}\right) \in X \times X^{*}$ with $f(x)<\infty$, one has

$$
\bar{\nabla}^{2} f\left(x, x^{*}\right)-L_{s}^{+}(X) \subset \bar{\partial}^{2} f\left(x, x^{*}\right) .
$$

If $f$ is continuous and paraconcave around $x$, equality holds.

Proof. Clearly if $H=\lim _{n} \nabla^{2} f\left(x_{n}\right)$, where $\left(x_{n}\right) \rightarrow x,\left(f\left(x_{n}\right)\right) \rightarrow f(x),\left(\nabla f\left(x_{n}\right)\right) \rightarrow$ $x^{*}$ and $\nabla^{2} f\left(x_{n}\right)$ exists, then $\nabla^{2} f\left(x_{n}\right) \in \partial_{-}^{2} f\left(x_{n}, x_{n}^{*}\right)$, where $x_{n}^{*}=f^{\prime}\left(x_{n}\right)$, so that $\left(x^{*}, H\right) \in \bar{\partial}^{2} f(x)$ or $H \in \bar{\partial}^{2} f\left(x, x^{*}\right)$.

Conversely, by the argument of [5], Lemma A4, rephrased in [17], Lemma 3.2, if $A \in \bar{\partial}^{2} f\left(x, x^{*}\right)$ one can find $H \in \bar{\nabla}^{2} f\left(x, x^{*}\right)$ with $A \leq H$. In fact, if $\left(x_{n}, x_{n}^{*}, A_{n}\right) \rightarrow$ $\left(x, x^{*}, A\right)$ with $\left(f\left(x_{n}\right)\right) \rightarrow f(x)$ and $\left(x_{n}^{*}, A_{n}\right) \in \partial_{-}^{2} f\left(x_{n}\right)$ for each integer $n$, then we can find $H_{n} \in \bar{\nabla}^{2} f\left(x_{n}, x_{n}^{*}\right)$ such that $A_{n} \leq H_{n} \leq \alpha I$ for some $\alpha>0$; taking a subsequence, we may assume $\left(H_{n}\right)$ converges to some $H \in \bar{\nabla}^{2} f\left(x, x^{*}\right)$.

The following result settles positively an open question in [7] (Remark 3.2).

Corollary 6.2. If $f$ is continuous and paraconcave around $x$ and attains a finite local minimum at $x$, then there exists $H \in \bar{\nabla}^{2} f(x, 0)$ with $H \geq 0$.

Proof. The result is a rewording of the inclusions

$$
0 \in \partial_{-}^{2} f\left(x, x^{*}\right) \subset \bar{\partial}^{2} f\left(x, x^{*}\right)=\bar{\nabla}^{2} f\left(x, x^{*}\right)-L_{s}^{+}(X) .
$$

The preceding result enables us to relate the limiting subhessians of a function which is both paraconvex and paraconcave, hence differentiable, with the generalized second order derivative given by

$$
f^{\circ \circ}(x ; u, v)=\limsup _{\substack{t \rightarrow 0+\\ w \rightarrow x}} t^{-1}\left[f^{\prime}(w+t u, v)-f^{\prime}(w, v)\right]
$$

(see [3] and [8]).

Proposition 6.3. Let $f: X_{0} \rightarrow \mathbb{R}$ be a function which is both paraconvex and paraconcave on an open subset $X_{0}$ of $X$. Then for any $x \in X$ and $v \in X$ one has, with $x^{*}=\nabla f(x)$,

$$
f^{\circ \circ}(x ; v, v)=\max \left\{(A v \mid v): A \in \bar{\partial}^{2} f\left(x, x^{*}\right)\right\} .
$$

Proof. By [6], $f$ is of class $C^{1,1}$, so that if one denotes by $\partial_{H}^{2} f(x)$ the convex hull of $\bar{\nabla}^{2} f(x):=\bar{\nabla}^{2} f\left(x, x^{*}\right)$ with $x^{*}=\nabla f(x)$, one has by [2], Theorem 2.1, for any $x \in X_{0}, v \in X$,

$$
\begin{aligned}
f^{\circ \circ}(x ; v, v) & =\max \left\{(A v \mid v): A \in \partial_{H}^{2} f(x)\right\} \\
& =\max \left\{(A v \mid v): A \in \bar{\nabla}^{2} f(x)\right\}
\end{aligned}
$$

since $A \rightarrow(A v \mid v)$ is linear on $L_{s}(X)$. Then the result follows from the preceding proposition.

Corollary 6.4 (second order Taylor's expansion). Let $f: X_{0} \rightarrow \mathbb{R}$ be a function which is both paraconvex and paraconcave on an open subset $X_{0}$ of $X$. Then for any $x, y$ in $X_{0}$ such that the segment $[x, y]$ is contained in $X_{0}$ there exist $z \in[x, y]$ and $A \in \bar{\partial}^{2} f\left(z, z^{*}\right)$ with $z^{*}=\nabla f(z)$ such that

$$
f(y)=f(x)+\left(z^{*} \mid y-x\right)+\frac{1}{2}(A(y-x) \mid y-x) .
$$


Proof. Let $r=2\left(f(y)-f(x)+f^{\prime}(x, v)\right)$, where $v=x-y$, so that the function $g:[0,1] \rightarrow \mathbb{R}$ given by

$$
g(t)=f(y)-f(y+t v)+t f^{\prime}(y+t v, v)-\frac{1}{2} r t^{2}
$$

satisfies $g(1)=0$ and $g(0)=0$. Since $f$ is of class $C^{1,1}, g$ is continuous. As $g(1)=g(0)$, we cannot have $D_{+} g(t)<0$ for each $t \in[0,1)$. Thus, there exists some $z=y+t v$ with $t \in[0,1)$ for which we have

$$
\begin{aligned}
0 & \leq \limsup _{s \rightarrow 0_{+}} s^{-1}(g(t+s)-g(t)) \\
& =t \limsup _{s \rightarrow 0_{+}} s^{-1}\left(f^{\prime}(z+s v, v)-f^{\prime}(z, v)\right)-r t
\end{aligned}
$$

hence

$$
r \leq f^{\circ \circ}(z, v, v)
$$

Proposition 6.3 ensures that for $z^{*}=f^{\prime}(z)$ we can find $A \in \bar{\partial}^{2} f\left(z, z^{*}\right)$ such that $r=(A v \mid v)$. Therefore the definition of $r$ yields the result.

Let us now introduce a notion of strict (or circa-)epi-derivative of order two and point out a link with limiting subhessians.

Recall that if $T$ and $W$ are metric spaces, if $q: T \times W \rightarrow \mathbb{R} \cup\{\infty\}$, and if $S$ is a subset of $T$, then for $r \in S, v \in W$ one sets

$$
\begin{aligned}
\limsup _{s \stackrel{S}{\rightarrow} r} \inf _{w \rightarrow v} q(s, w) & =\sup _{V \in \mathcal{N}(v)} \inf _{R \in \mathcal{N}(r)} \sup _{s \in R \cap S} \inf _{w \in V} q(s, w) \\
& =\sup _{\left(r_{n}\right) \stackrel{S}{\rightarrow} r} \inf _{\left(v_{n}\right) \rightarrow v} \limsup _{n} f\left(r_{n}, v_{n}\right) .
\end{aligned}
$$

With this in mind let us set

$$
f^{\uparrow \uparrow}\left(x, x^{*}, v\right)=\limsup _{\substack{\left.t \rightarrow 0 \\\left(u, f(u), u^{*}\right) \stackrel{J f}{\rightarrow}\left(x, f(x), x^{*}\right)\right)}} \inf _{w \rightarrow v} 2 t^{-2}\left[f(u+t w)-f(u)-\left(u^{*} \mid t w\right)\right],
$$

with

$$
J f=\left\{\left(x, r, x^{*}\right) \in X \times \mathbb{R} \times X^{*}: r=f(x), x^{*} \in \partial_{-} f(x)\right\} .
$$

Clearly one has

$$
f_{-}^{\prime \prime}\left(x, x^{*}, v\right) \leq f^{\uparrow \uparrow}\left(x, x^{*}, v\right),
$$

but this inequality does not suffice to prove the following result.

Proposition 6.5. For any $x \in \operatorname{dom} f, x^{*} \in \partial f(x), v \in X$ and $A \in \bar{\partial}^{2} f\left(x, x^{*}\right)$ one has

$$
(A v \mid v) \leq f^{\uparrow \uparrow}\left(x, x^{*}, v\right) .
$$

Proof. For $A \in \bar{\partial}^{2} f\left(x, x^{*}\right)$ we can find a sequence $\left(x_{n}, x_{n}^{*}, A_{n}\right)$ with limit $\left(x, x^{*}, A\right)$ such that $\left(f\left(x_{n}\right)\right) \rightarrow f(x)$ and $\left(x_{n}^{*}, A_{n}\right) \in \partial_{-}^{2} f\left(x_{n}\right)$ for each $n$. Hence

$$
\left(x_{n}, f\left(x_{n}\right), x_{n}^{*}\right) \stackrel{J f}{\rightarrow}\left(x, f(x), x^{*}\right) .
$$

Given $\varepsilon>0$, for each $n \in \mathbb{N}$ we can find $\rho_{n}>0$ such that

$$
f\left(x_{n}+z\right) \geq f\left(x_{n}\right)+\left(x_{n}^{*} \mid z\right)+\frac{1}{2}\left(A_{n} z \mid z\right)-\varepsilon\|z\|^{2}
$$


for $\|z\| \leq \rho_{n}$. Taking $t_{n}=\min \left(2^{-n},(2\|v\|)^{-1} \rho_{n}\right)$ so that for any sequence $\left(v_{n}\right)$ with limit $v$ we have $\left\|t_{n} v_{n}\right\| \leq \rho_{n}$ for $n$ large enough, we have

$$
2 t_{n}^{-2}\left[f\left(x_{n}+t_{n} v_{n}\right)-f\left(x_{n}\right)-\left(x_{n}^{*} \mid t_{n} v_{n}\right)\right] \geq\left(A_{n} v_{n} \mid v_{n}\right)-2 \varepsilon\left\|v_{n}\right\|^{2},
$$

so that

$$
\limsup _{n} 2 t_{n}^{-2}\left[f\left(x_{n}+t_{n} v_{n}\right)-f\left(x_{n}\right)-\left(x_{n}^{*} \mid t_{n} v_{n}\right)\right] \geq(A v \mid v)-2 \varepsilon\|v\|^{2} .
$$

Taking the infimum over $\left(v_{n}\right)$ and then the supremum over $\left(x_{n}, x_{n}^{*}, t_{n}\right) \rightarrow\left(x, x^{*}, 0\right)$ and $\varepsilon>0$, we get

$$
f^{\uparrow \uparrow}\left(x, x^{*}, v\right) \geq(A v \mid v)
$$

\section{REFERENCES}

[1] J.-P. Aubin and H. Frankowska, Set-Valued Analysis, Birkhäuser, Basel, 1990. MR 91d:49001

[2] R. Cominetti and R. Correa, Sur une dérivée du second ordre en analyse non différentiable, C. R. Acad. Sci. Paris Sér. I Math. 303 (1986), 861-864. MR 87m:49040

[3] _ A generalized second-order derivative in nonsmooth optimization, SIAM J. Control Optim. 28 (4) (1990), 789-809. MR 91h:49017

[4] M. G. Crandall and H. Ishii, The maximum principle for semicontinuous functions, Differential and Integral Equations 3 (1990), 1001-1014.

[5] M. G. Crandall, H. Ishii, and P.-L. Lions, User's guide to viscosity solutions of second order partial differential equations, Bull. Amer. Math. Soc. (N.S.) 27 (1992), 1-67. MR 92j:35050

[6] J.-B. Hiriart-Urruty and Ph. Plazanet, Moreau's theorem revisited, Analyse Non Linéaire (Perpignan, 1987), Ann. Inst. H. Poincaré Anal. Non Linéaire 6 (1989), suppl., 325-338. MR 91b:58017

[7] J.-B. Hiriart-Urruty, J.-J. Strodiot and V. H. Nguyen, Generalized hessian matrix and second order conditions for problems with $C^{1,1}$ data, Appl. Math. Optim. 11 (1984), 43-56. MR 85g:49031

[8] A. D. Ioffe, Second order conditions in nonlinear nonsmooth problems of semi-infinite programming, Semi-infinite Programming and Applications, Lecture Notes in Economics and Math. Systems, vol. 215, Springer-Verlag, Berlin, 1983, pp. 262-280. MR 84m:49060

[9] _ Approximate subdifferentials and applications. 1: The finite dimensional theory, Trans. Amer. Math. Soc. 281 (1984), 389-416; 288 (1985), 429. MR 84m:49029; MR 86d:49023

[10] _ Proximal analysis and approximate subdifferentials, J. London Math. Soc. 41 (1990), 175-192. MR 91i:46045

[11] R. Jensen, The maximum principle for viscosity solutions of fully nonlinear second order partial differential equations, Arch. Rational Mech. Anal. 101 (1988), 1-27. MR 89a:35038

[12] A. Ya. Kruger, Properties of generalized differentials, Sibirsk. Mat. Zhurnal 26 (1985), no. 6, 54-66; English transl., Siberian Math. J. 26 (1985), 822-832. MR 87d:90147

[13] L. A. Lusternik and V. J. Sobolev, Elements of functional analysis, Hindustan, Delhi, and Wiley, New York, 1974. MR 50:2852

[14] B. Sh. Mordukhovich, Maximum principle in the problem of time-optimal response with nonsmooth constraints, J. Appl. Math. Mech. 40 (1976), 960-969. MR 58:7284

[15] _ Nonsmooth analysis with nonconvex generalized differentials and adjoint mappings, Dokl. Akad. Nauk BSSR 28 (1984), 976-979. (Russian) MR 86c:49018

[16] J.-P. Penot, Regularity conditions in mathematical programming, Math. Programming Study 19 (1982), 167-199. MR 84d:90095

[17] _ Subhessians, superhessians and conjugation, Nonlinear Anal. 23 (1994), 689-702. MR 95h:49025

[18] J. P. Penot and M. Volle, On strongly convex and paraconvex dualities, Generalized Convexity and Fractional Programming with Economic Applications, A. Cambini et al. (eds.), Lecture Notes in Economics and Math. Systems, vol. 345, Springer-Verlag, Berlin, 1990, pp. 198-218. MR 92h: 49041 
[19] R. T. Rockafellar, Favorable classes of Lipschitz continuous functions in subgradient optimization, Progress in Nondifferentiable Optimization (E. Nurminski, ed.), IIASA Collaborative Proc. Ser. CP-82, 8, Internat. Inst. Appl. Systems Analysis, Laxenburg, Austria, 1982, pp. 125-144. MR 85e:90069

[20] _ First- and second-order epi-differentiability in nonlinear programming, Trans. Amer. Math. Soc. 307 (1988), 75-108. MR 90a:90216

[21] S. Rolewicz, On paraconvex multifunctions, Proc. III Sympos. Oper. Research, Mannheim, 1978, 539-546. MR 80i:49025

Department of Mathematics, Technion, 32000 Haifa, Israel

Départment de Mathématiques, CNRS URA 1204, Faculté des Sciences, Av. De L’UniVERsité, 64000 Pau, France

E-mail address: jean-paul.penot@univ.pau-fr 